\title{
Metachronous urothelial carcinoma of whole urinary tract in a dialysis-dependent patient: A case report
}

\author{
YIMIN WANG, BAIYE JIN and XIAOLIN YAO \\ Department of Urology, The First Affiliated Hospital, School of Medicine, \\ Zhejiang University, Hangzhou, Zhejiang 310003, P.R. China
}

Received March 17, 2015; Accepted April 8, 2016

DOI: $10.3892 / \mathrm{ol} .2016 .4537$

\begin{abstract}
Numerous studies have reported an association between end-stage renal disease (ESRD) and malignancy. The predominant malignant tumor that occurs in patients with ESRD in Asian countries is urothelial carcinoma (UC). According to recent research, cases of UC in dialysis-dependent patients are associated with higher recurrence rates and more aggressive biological behavior compared with patients without ESRD. The necessity of 1-step total urinary tract exenteration for dialysis-dependent patients with UC is advocated by certain studies. The current study reports a case of metachronous bladder cancer and bilateral upper urinary tract tumor in a dialysis-dependent patient. Three separate surgeries were performed to remove the bladder, and left and right urinary tract, respectively. The question of whether the stepwise strategy or the 1-step surgery should be selected for such special cases remains in debate.
\end{abstract}

\section{Introduction}

Increasing epidemiological data indicate a growing relative risk of malignancy among patients with end-stage renal disease (ESRD), including renal cell carcinoma (RCC), urothelial carcinoma (UC), and thyroid and lung cancer (1). The predominant tumor in Western countries is RCC, while it is UC in Asian countries (2). Clinically, UC is a multifocal malignant disease located in the uroepithelium. Recent data demonstrate that $2-4 \%$ of patients with a bladder UC also present with an upper urinary tract tumor (UUTT); the incidence of UUTT is 2-6\% among patients who undergo a radical cystectomy for UC (3). Higher recurrence rates and more aggressive biological behavior of UC in dialysis-dependent patients have been confirmed compared with individuals

Correspondence to: Dr Xiaolin Yao, Department of Urology, The First Affiliated Hospital, School of Medicine, Zhejiang University, 79 Qingchun Road, Hangzhou, Zhejiang 310003, P.R. China E-mail: yx1_1xy@126.com

Key words: metachronous neoplasms, dialysis, bladder cancer, pelvic neoplasms, ureteral neoplasms without ESRD (2). Additionally, these cases of UC are not easy to monitor in dialysis-dependent patients due to oliguria or anuria, which may obscure the typical symptoms, such as gross hematuria (4).

The current study presents a case of metachronous bladder cancer and bilateral UUTT in a dialysis-dependent patient who received total cystectomy and left and right urinary tract exenteration surgeries within 18 months.

\section{Case report}

A 35-year-old woman with end-stage renal failure owing to nephrotoxic drugs had been on dialysis for three years, since October 2011, in The First Affiliated Hospital of Zhejiang University School of Medicine (Hangzhou, China). The patient had experienced gross hematuria for 1 year and had been diagnosed with a bladder tumor by ultrasound (MyLab Twice; Esaote Group, Milano, Italy) (Fig. 1A) and cystoscope 1 month previously. The mass was located in anterior wall and measured $2.3 \times 2.7 \mathrm{~cm}$. Pathological analysis of a biopsy specimen was conducted. Hematoxylin and eosin staining of the paraffin-embedded section revealed that the tumor tissue exhibited cellular atypia and nuclear abnormity, and karyokinesis could be easily observed. Infiltrative growth of the malignant cells indicated aggressive biological behavior. These findings suggested a highly malignant lesion. Total cystectomy was performed following admission of the patient to our hospital. The final pathological diagnosis was high grade invasive UC accompanied by sarcomatoid carcinoma (Fig. 1B). The surgery was successful, and there was no sign of recurrence on regular computed tomography (Brilliance 64; Royal Dutch Philips Electronics, Ltd., Amsterdam, Netherlands) postoperatively.

However, 6 months later, a 2.2-cm space-occupying lesion in the right pelvis was detected by ultrasound (Fig. 2A), which was indicated to be a malignant tumor. According to standard principles, a right urinary tract exenteration was selected as curative treatment for the patient, and high-grade invasive UC was subsequently confirmed by pathologists (Fig. 2B). Postoperatively, dialysis was continued for renal replacement therapy (RRT).

After another 6 months, multiple left pelvis and ureteral tumors were identified by ultrasound (Fig. 3A); the largest tumor was detected in the pelvis and had a diameter of 
A

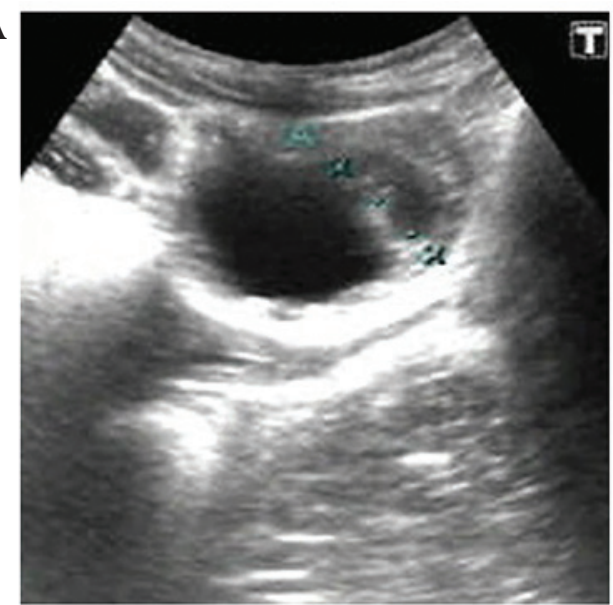

B

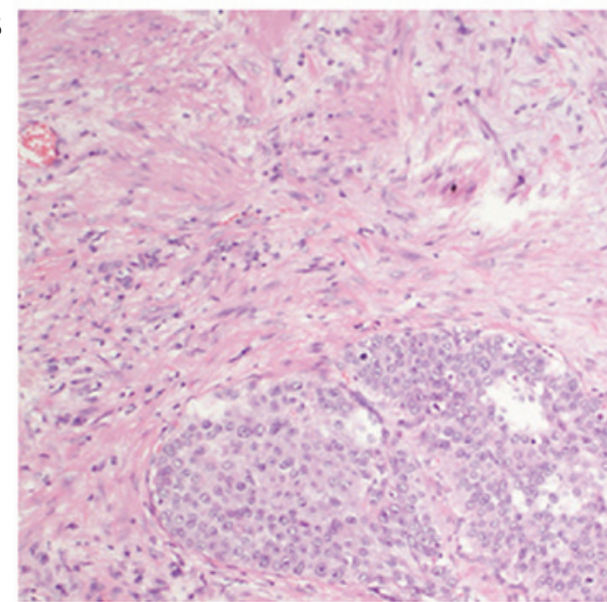

Figure 1. Bladder tumor findings. (A) Ultrasound image and (B) histopathological image (hematoxylin and eosin staining; magnification, x100). The tumor was diagnosed as high-grade invasive urothelial carcinoma.
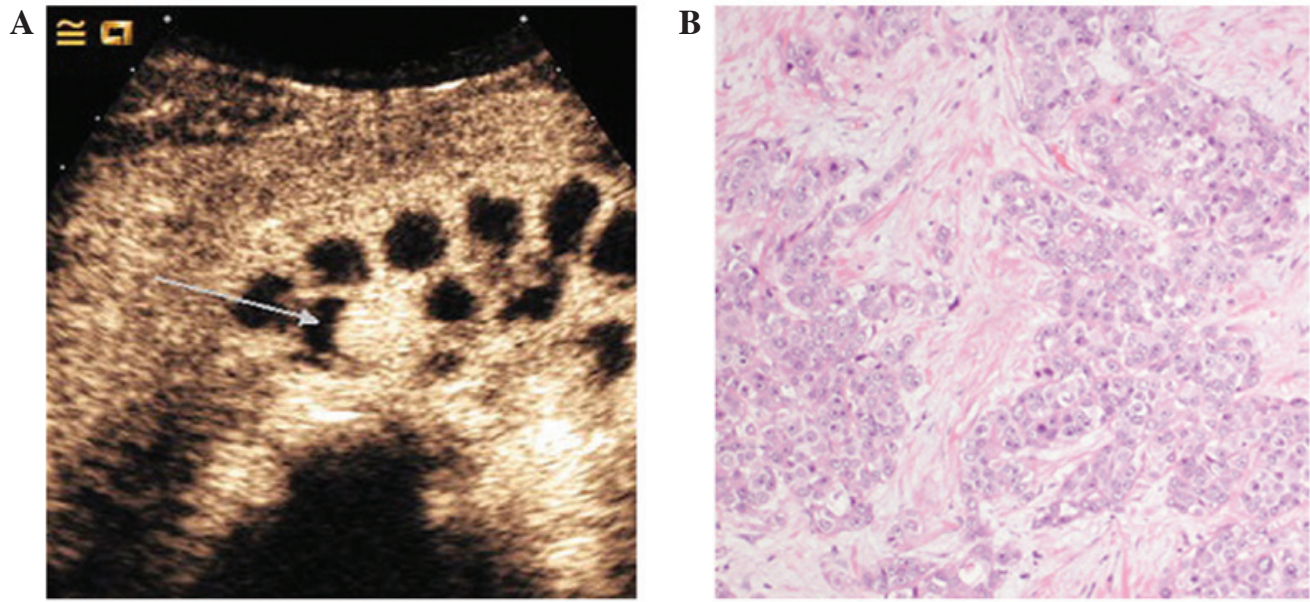

Figure 2. Right pelvic tumor findings. (A) Ultrasound image and (B) histopathological image (hematoxylin and eosin staining; magnification, x100). The tumor was diagnosed as high-grade invasive urothelial carcinoma.
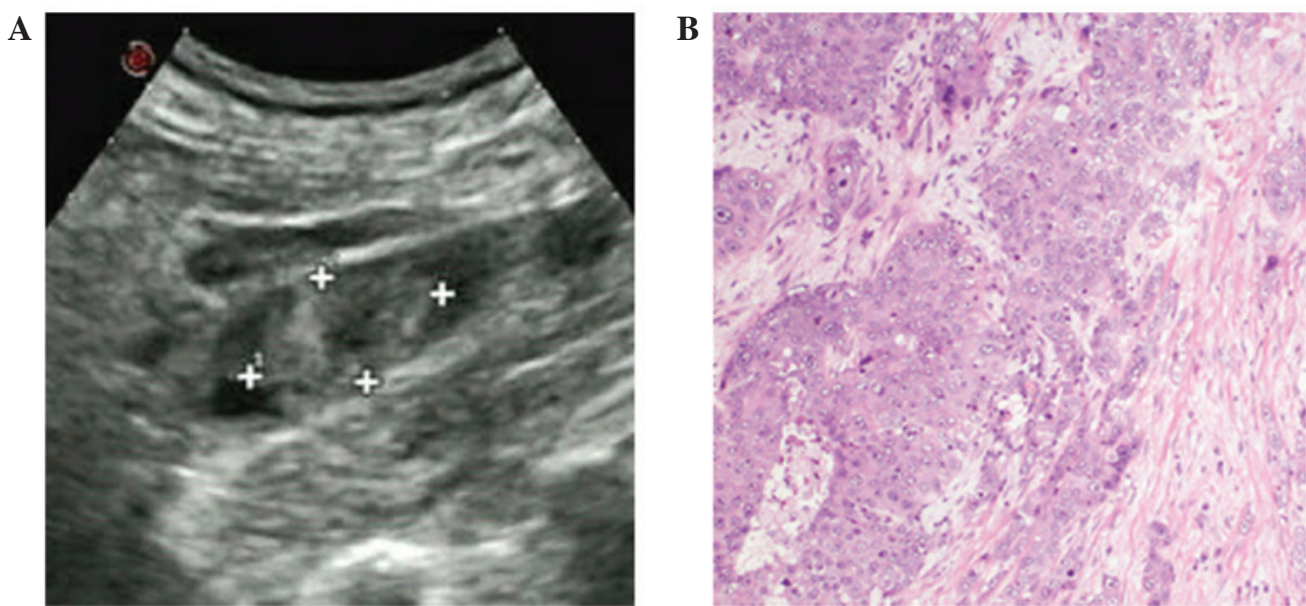

Figure 3. Left ureteral tumor findings. (A) Ultrasound image and (B) histopathological image (hematoxylin and eosin staining; magnification, x100). The tumor was diagnosed as high-grade invasive urothelial carcinoma.

$3.5 \mathrm{~cm}$. Left urinary tract exenteration was performed as required. High-grade invasive UC was eventually diagnosed (Fig. 3B). At this point, the whole urinary tract of the patient had been removed via three separate surgeries. Continuous dialysis was administered without interruption during the surgeries. No recurrence was detected in the patient for 
6 months following the final surgery. The patient was scheduled to receive regular follow-ups and dialysis.

Informed consent for the present report was obtained from the patient.

\section{Discussion}

In China, the variety of malignancies occurring among the population of patients undergoing dialysis is distinct from that in Western countries; the incidence of UC is more notable than any other type of malignancy among Chinese ESRD patients $(2,4,5)$, whilst renal cell carcinoma is more common in Western countries. The reason for the high incidence and prevalence of UC in ESRD patients in China is unknown; however, chronic bladder irritation, decreased urinary washout effect, and atrophic involution of the bladder may be contributing factors. In the current case, the patient received dialysis for RRT, and experienced metachronous UC of the entire urinary tract, which was similar to the results of the previous studies $(2,4,5)$. Surgical excision is the preferred treatment in this circumstance.

Currently, maintenance dialysis is one of predominant therapies for ESRD patients worldwide (1). However, the influence of dialysis on the characteristics of UC in the RRT population remains uncertain. Numerous studies have focussed on elucidating the association between UC and dialysis (4,6-8). The etiology of UC in patients undergoing RRT is multifactorial and complex. Recent studies suggested that direct DNA damage by immunosuppression is important in ESRD patients. The association between UC and viral infection, however, remains a controversial topic $(6,7)$. On the other hand, drug-induced immunosuppression following renal transplant, and chronic bladder irritation due to anuria in ESRD patients, which decreases the effect of urinary tract washing by urine flow, have been reported to be predisposing factors for UC development (4). Additionally, epidemiological investigations indicate that the factors involved in the development of UC in RRT patients may include smoking, analgesic nephropathy, arsenic poisoning, and uremia (8).

With regard to the treatment of UC, this may differ between dialysis patients and the normal population; a more aggressive approach may be selected for ESRD patients due to the higher rate of recurrence and metachronous UC. Total urinary tract exenteration may be an optional therapeutic strategy for this specific population due to non-functional urinary tracts, but this is not available for patients without ESRD. In the current case, metachronous UC of whole urinary tract required three major surgeries, which resulted in severe physiological and psychological effects on the patient. As dialysis patients with UC have a higher rate of recurrence and metachronous tumors than non-ESRD patients, it is possible that total urinary tract exenteration may be a good therapeutic option for this specific population. A recent study of dialysis patients with UC reported that the 5-year survival rate of patients undergoing radical cystectomy with concurrent nephroureterectomy was better compared with that of patients who did not undergo concurrent nephroureterectomy. The authors suggest aggressive treatment in patients in a chronic stable condition to improve survival rate (9). However, a high mortality rate was noted in patients undergoing cystectomy with concurrent nephroureterectomy due to various complications $(9,10)$. This topic remains in controversial. For the high-risk, dialysis-dependent ESRD patient group, at least, total urinary tract exenteration could be a good therapeutic option due to the high incidence of tumors in the non-functioning upper and lower urinary tract.

In summary, the current report presents a case of metachronous UC of whole urinary tract in a dialysis-dependent patient, which indicates the more aggressive features of UC in this specific population. A surgical approach is the first choice for these patients. Total urinary tract exenteration is an available option; however, the status of the patient and the stage of the renal disease should be considered prior to surgery.

\section{Acknowledgements}

The current study was supported by a grant from the Scientific Research Fund of the Health Bureau of Zhejiang Province (grant no. 2015KYA072).

\section{References}

1. Weng PH, Hung KY, Huang HL, Chen JH, Sung PK and Huang KC: Cancer specific mortality in chronic kidney disease: Longitudinal follow-up of a large cohort. Clin J Am Soc Nephrol 6: 1121-1128, 2011.

2. Feng WW, Wang TN, Chen HC, Ho JC and Ko YC: Malignancies after renal transplantation in southern Taiwan: Experience in one centre. BJU Int 99: 825-829, 2007.

3. Izquierdo L, Truán D, Alvarez-Vijande R and Alcaraz A: Large series of 114 cases with long term follow-up of upper urinary tract urothelial tumors. Actas Urol Esp 34: 232-237, 2010 (In Spanish).

4. Ou JH, Pan CC, Lin JS, Tzai TS, Yang WH, Chang CC, Cheng HL, Lin YM and Tong YC: Transitional cell carcinoma in dialysis patients. Eur Urol 37: 90-94, 2000.

5. Wu MJ, Lian JD, Yang CR, Cheng $\mathrm{CH}$, Chen $\mathrm{CH}$, Lee WC, Shu KH and Tang MJ: High cumulative incidence of urinary tract transitional cell carcinoma after kidney transplantation in Taiwan. Am J Kidney Dis 43: 1091-1097, 2004.

6. Pino L, Rijo E, Nohales G, Frances A, Ubre A and Arango O: Bladder transitional cell carcinoma and BK virus in a young kidney transplant recipient. Transpl Infect Dis 15: E25-E27, 2013.

7. Xiao J, Zhu X, Hao GY, Zhu YC, Ma LL, Zhang YH and Tian Y: Association between urothelial carcinoma after renal transplantation and infection by human papillomavirus types 16 and 18 . Transplant Proc 43: 1638-1640, 2011.

8. Zhang B, Shen C, Han WK and Yu W: Comparison of clinicopathologic characteristics of urothelial carcinoma between patients after renal transplantation and on dialysis. Transplantation 98: 552-556, 2014.

9. Tseng SF, Chuang YC and Yang WC: Long-term outcome of radical cystectomy in ESDR patients with bladder urothelial carcinoma. Int Urol Nephrol 43: 1067-1071, 2011.

10. Wu CF, Shee JJ, Ho DR, Chen WC and Chen CS: Different treatment strategies for end stage renal disease in patients with transitional cell carcinoma. J Urol 171: 126-129, 2004 